\title{
Bark polyflavonoids from Pinus radiata as functional building-blocks for polylactic acid (PLA)-based green composites
}

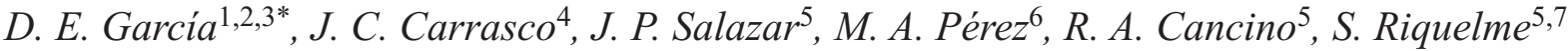 \\ ${ }^{1}$ Laboratorio de Fitoquímica, Departamento de Química Ambiental, Facultad de Ciencias, Universidad Católica de la \\ Santísima Concepción (UCSC), Concepción, Biobío, Chile \\ ${ }^{2}$ Centro de Investigación en Biodiversidad y Ambientes Sustentables (CIBAS), UCSC, Concepción, Biobío, Chile \\ ${ }^{3}$ Investigador Asociado Área de Productos Químicos, Unidad de Desarrollo Tecnológico (UDT), Universidad de \\ Concepción, Concepción, Biobío, Chile \\ ${ }^{4}$ Área de Biomateriales, UDT, Universidad de Concepción, Concepción, Biobío, Chile \\ ${ }^{5}$ Área de Productos Químicos, UDT, Universidad de Concepción, Concepción, Biobío, Chile \\ ${ }^{6}$ Departamento de Polímeros, Facultad de Ciencias Químicas, Universidad de Concepción, Concepción, Chile \\ ${ }^{7}$ Programa de Doctorado en Ciencias y Tecnologías Analíticas, Universidad de Concepción, Concepción, Biobío, Chile
}

Received 1 April 2016; accepted in revised form 22 May 2016

\begin{abstract}
Polylactic acid (PLA) was melt-blended with Pinus radiata unmodified and modified (hydroxypropyled) bark polyflavonoids in order to use such polyphenolic building-blocks as functional additives for envisaged applications. Rheological, morphological, molecular, thermal, and flexural properties were studied. Polyflavonoids improved blend processability in terms of short-time mixing. Furthermore, hydroxypropylated polyflavonoids improve miscibility in binary and ternary blends. Blend-composition affects crystallization-, melting-, and glass transition-temperature of PLA, as well as thermal resistance, and flexural properties of the blends. Polyflavonoids induced PLA-crystallization, and polymer-chain decomposition. Modified and unmodified bark polyflavonoids from radiata pine can be used successfully in PLA-based green composites beyond the food-packaging applications. The high compatibility between PLA and hydroxypropyled polyflavonoids highlights the potential of such phenolic derivatives for PLA-based material design.
\end{abstract}

Keywords: polymer composites, hydroxypropyl polyflavonoid, radiata pine, polymer processability

\section{Introduction}

The most important polymers in bioplastic engineering are aliphatic polyesters such as polylactic acid (PLA) [1]. Blending of PLA with other polymers such as polypropylene, natural rubbers, polyglycols (PEG), polyvinyl acetate, polyolefins, polymethyl methacrylate, and polycarbonate is an useful route towards modifying properties [2]. In addition, oligomers/polymers from lignocellulosic resources have been used as additives in order to reduce cost [3-5]. In fact, many advantages are recognized in binary and ternary blends regarding well dispersion into the PLA-matrix, and the nucleating effect of certain biopolymers which induced dramatic changes on mechanical properties [6].

Moreover, lignin and condensed tannin (polyflavonoids) have been successfully blended with PLA [7]. However, there are few reports which describe the effect of polyflavonoids on the PLA physicochemical properties. Polyphenols properties such as an- 
tioxidant capacity, strong UV-absorption, and remarkable biological activity (antibacterial/antifungal) enables utilizing polyflavonoids as functional buildingblocks for PLA-based composites. Such selected properties might be considered in order to design functional materials based on key polyphenols properties such as $\mathrm{O}_{2}$-active barrier for food packaging, UV-filter for selected outdoor applications, antimicrobial surfaces for food industry, as well as functional films and plastic devices for agriculture.

On the other hand, polyflavonoids are highly abundant in bark that is expected to play an important role in bioplastics engineering, and thermosetting systems $[8,9]$. However, limited solubility in organic solvents and molten thermoplastics, and low thermal resistance of polyflavonoids are well-known drawbacks for polymer formulation, that is why chemical modification with alkylene oxides enabling less polar derivatives highly desirable for biomaterial design [10]. Hydroxypropyl polyflavonoids (HPT, Figure 1) from pine bark is easily synthesized at room temperature with propylene oxide, and the versatility as macro building-blocks has been demonstrated in several chemistries [11].

This work reports the preparation and characterization of several PLA-based blends using bark polyflavonoids (with and without chemical modification) as the main functional additive. Rheological, morphological, molecular, thermal, and flexural analyses were performed. The new types of PLA-based coloured materials are expected to play an important role beyond the traditional food-packaging applications of PLA.

\section{Materials and methods}

\subsection{Chemicals}

\subsubsection{Polylactic acid (PLA)}

Partially crystalline PLA (Polylactic acid) of Natureworks Ingeo ${ }^{\mathrm{TM}}$ Biopolymer 3251 grade supplied by NatureWorks ${ }^{\circledR}$ Co. LLC, USA, was the thermoplastic used in this study: average $M_{\mathrm{w}} 90000-150000$ Dalton, polydispersity $(D) 1.6$, melting tempetarure $\left(T_{\mathrm{m}}\right)$ $170^{\circ} \mathrm{C}$, glass transition temperature $\left(T_{\mathrm{g}}\right) 61^{\circ} \mathrm{C}$, and L-lactide content of $98 \%$.

\subsubsection{Polyflavonoid}

Non-water soluble radiata pine bark polyflavonoids (PRI) extracted with methanol/water solution (1:20, $\mathrm{w} / \mathrm{v})$ at $120^{\circ} \mathrm{C}$ was used [12]. Briefly, PRI are represented by a broad mixture of polyphenols ( $D: 1.5-$ 2.8 ) mostly $4 \rightarrow 8$ linked $[13,14]$.

\subsubsection{Hydroxypropyl polyflavonoid (PRI-3)}

PRI-3 was synthesized by open-ring reaction $\left(S_{\mathrm{N}} 2\right)$ using propylene oxide (PO) in alkali media ( $\mathrm{pH}: 12)$ at room temperature $\left(20-22^{\circ} \mathrm{C}\right)$ as described by García et al. [8]. The parameters of the derivatization were described in detail elsewhere [9].

Briefly, PRI (100 g, ca. $80 \mathrm{mmol})$ was dissolved in $500 \mathrm{~mL}$ aq. 2 molL-1 $\mathrm{NaOH}$, adjusted to $\mathrm{pH} 12$ and stoichiometric PO-charge added. The reaction was carried out for $24 \mathrm{~h}$ with stirring. Before that, $\mathrm{HCl}$ $(40 \% \mathrm{v} / \mathrm{v})$ was added to adjust the $\mathrm{pH}$ to 2 , producing a precipitate that was centrifuged. The supernatant was collected by decantation and the precipitate was washed five times with distilled water and freeze-

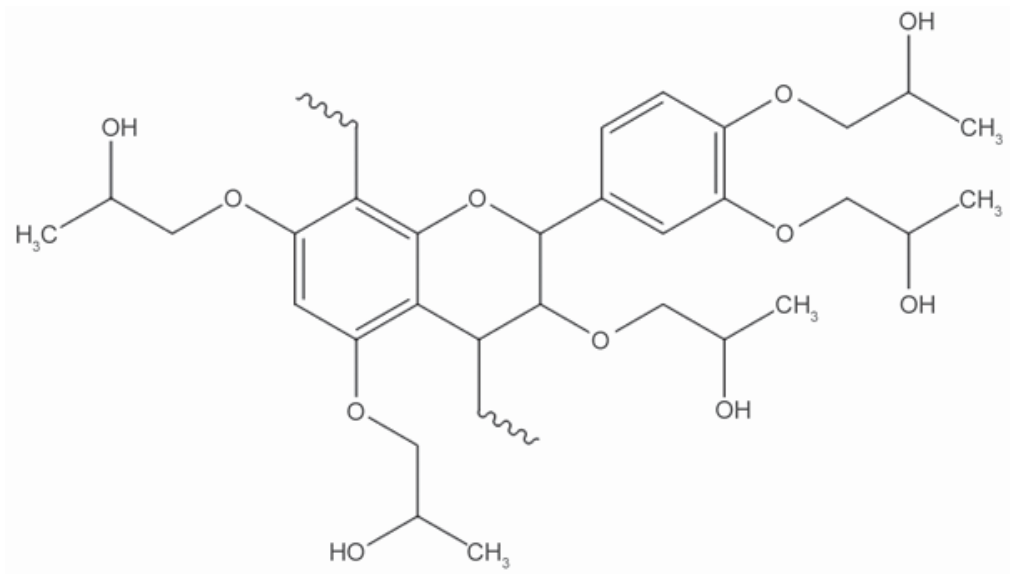

Figure 1. Chemical structure of hydroxypropyl polyflavonoid $\left(\mathrm{C}_{15}\right.$ unit fully modified by hydroxypropylation) 
dried. Reaction yields ranged from $82-90 \mathrm{wt} \%$ and the substitution degree (DS) was determined by ${ }^{1} \mathrm{H}-\mathrm{NMR}$ (DS: $3.0 \pm 0.2$ ).

\subsubsection{Plasticizer}

CARBOWAX $^{\text {TM }}$ (polyethylene glycol- $M_{\mathrm{w}} 400 \mathrm{~g} \cdot \mathrm{mol}^{-1}$ ) supplied by Dow Chemical Company (Chile) was used as a conventional plasticizer. Technical specifications are described as follow: density: $1.13 \mathrm{~g} \cdot \mathrm{cm}^{-3}$, viscosity $\left(100^{\circ} \mathrm{C}\right): 7.3 \mathrm{mPa} \cdot \mathrm{s}$, average number of repeating oxyethylene units $(n): 8.7$.

\subsection{Blends formulation}

Blends based on PLA were prepared at $170{ }^{\circ} \mathrm{C}$ for $15 \mathrm{~min}$ in a torque rheometer (Brabender $50 \mathrm{EHT}$, Germany). Binary blends were prepared in order to understand the effect of components on physicochemical properties (Table 1). Ternary blends were formulated with modified and unmodified polyflavonoids $(10,20,30 \mathrm{wt} \%)$, as well as PEG.

\subsection{Preparation of the blends}

Solid components (PLA, PRI, and PRI-3) were pretreated by oven-drying at $60^{\circ} \mathrm{C}$ for $72 \mathrm{~h}$ to eliminate possible absorbed water on the surface of the particles. In a specific order components were added in a rheometer. PLA and polyflavonoids were mixed for $2 \mathrm{~min}$ in a mixer (mini SWJB Lab mixing equipment, China), and loaded into the chamber (time mixing, $t_{\text {mix }}: 0$ ). In ternary blends, PEG was loaded after the PLA-melting ( $t_{\text {mix }}: 1.5$ min). Three blends of each formulation were prepared.

\subsubsection{Cold-blend processing}

Blends were ground in an electric mill (IKA, Basic MF10, China) and $30 \mathrm{~g}$ charges were compression moulded on a LabTech LP20-B press $\left(T: 175^{\circ} \mathrm{C}, t\right.$ : $5 \mathrm{~min}, p$ : 32 bar). Laminates of $100 \mathrm{~mm}^{2}$ (thickness: $1.5 \mathrm{~mm}$ ) were used for the flexural, and microscopy testing.

\subsection{Physicochemical properties}

\subsubsection{Rheology}

Rheology measurements were carried out in a torque rheometer with roller blades. The rotor speed was $50 \mathrm{rpm}$, and the free volume of the chamber was $20 \%$ of the total volume.

\subsubsection{Confocal microscopy (particle size distribution)}

Particle morphology of the blends was assessed by confocal fluorescence microscopy.

The fluorescense emission pattern of polyflavonoids (powder), and blends (laminate) were assessed by a systematic analysis. Dry samples were irradiated (Laser I: $\lambda_{405 \mathrm{~nm}}$, Laser II: $\lambda_{488 \mathrm{~nm}}$, and Laser III: $\lambda_{561 \mathrm{~nm}}$ ), and fluorescence emission recovered. A Zeiss LSM 780 confocal microscope with several diode laser and a Coherent Chameleon laser (Ti:sapphire) for onephoton, was used in the experiments. Samples were

Table 1. Composition of PLA-based blends prepared at $170^{\circ} \mathrm{C}$

\begin{tabular}{|c|c|c|c|c|c|}
\hline \multirow[t]{2}{*}{ Blend } & \multicolumn{4}{|c|}{$\begin{array}{c}\text { Component } \\
\text { [wt } \%]\end{array}$} & \multirow[t]{2}{*}{ Code } \\
\hline & PLA & PRI & PRI-3 & PEG & \\
\hline \multirow{3}{*}{ PLA/PRI } & 80 & 20 & 0 & 0 & $\mathrm{PLA}_{80} / \mathrm{PRI}_{20}$ \\
\hline & 70 & 30 & 0 & 0 & $\mathrm{PLA}_{70} / \mathrm{PRI}_{30}$ \\
\hline & 60 & 40 & 0 & 0 & $\mathrm{PLA}_{60} / \mathrm{PRI}_{40}$ \\
\hline \multirow{3}{*}{ PLA/PRI-3 } & 80 & 0 & 20 & 0 & $\mathrm{PLA}_{80} / \mathrm{PRI}-3_{20}$ \\
\hline & 70 & 0 & 30 & 0 & $\mathrm{PLA}_{70} / \mathrm{PRI}-3_{30}$ \\
\hline & 60 & 0 & 40 & 0 & $\mathrm{PLA}_{60} / \mathrm{PRI}-3_{40}$ \\
\hline \multirow{3}{*}{ PLA/PEG } & 80 & 0 & 0 & 20 & $\mathrm{PLA}_{80} / \mathrm{PEG}_{20}$ \\
\hline & 70 & 0 & 0 & 30 & $\mathrm{PLA}_{70} / \mathrm{PEG}_{30}$ \\
\hline & 60 & 0 & 0 & 40 & $\mathrm{PLA}_{60} / \mathrm{PEG}_{40}$ \\
\hline \multirow{3}{*}{ PLA/PRI/PEG } & 60 & 10 & 0 & 30 & $\mathrm{PLA}_{60} / \mathrm{PRI}_{10} / \mathrm{PEG}_{30}$ \\
\hline & 60 & 20 & 0 & 20 & $\mathrm{PLA}_{60} / \mathrm{PRI}_{20} / \mathrm{PEG}_{20}$ \\
\hline & 60 & 30 & 0 & 10 & $\mathrm{PLA}_{60} / \mathrm{PRI}_{30} / \mathrm{PEG}_{10}$ \\
\hline \multirow{3}{*}{ PLA/PRI-3/PEG } & 60 & 0 & 10 & 30 & $\mathrm{PLA}_{60} / \mathrm{PRI}-3_{10} / \mathrm{PEG}_{30}$ \\
\hline & 60 & 0 & 20 & 20 & $\mathrm{PLA}_{60} / \mathrm{PRI}-3_{20} / \mathrm{PEG}_{20}$ \\
\hline & 60 & 0 & 30 & 10 & $\mathrm{PLA}_{60} / \mathrm{PRI} 3_{30} / \mathrm{PEG}_{10}$ \\
\hline
\end{tabular}

PLA: poly-lactic acid, PRI: unmodified radiada pine polyflavonoid, PRI-3: hydroxypropyl radiata pine polyflavonoid (DS: 3), PEG: polyethylene glycol 
observed with a C-Apochromat objective lens (63×, numerical aperture 1.2) in the opposite side of the cover slip. The images were obtained by the average of two scans. In all experiments, at least three sites of the blend were studied, and no appreciable variation was observed in the fluorescence properties among or within-sample. Considering the numerical aperture and the wavelength of excitation, the spatial resolution was approximately $200 \mathrm{~nm}$. The optical zoom was $63 \times$. A further digital zoom was used. The percentages of the lasers nominal powers were $1.2 \%$ $(\sim 20 \mu \mathrm{W})$ for a $20 \times$ objective.

Image-analysis of the maximum intensity projection was used in order to estimate the polyflavonoid particle size distribution as a referential parameter of component miscibility. IMARIS software (version 7.5.2, module Pro program) allowed estimating various types of numerical values based on the three-dimensional structures in confocal images.

Volume calculation of polyflavonoid particles was carried out using an automatic segmentation tool (Spots). The following parameters were considered: (1) different sizes, (2) estimated diameter, (3) quality above automatic threshold, (4) region growing -absolute intensity threshold with 7000 (for red channel) and 7500 (for green channel)-, and (5) diameter from volume. The segmented particles volume values of each image were plotted in a normalized histogram (in logarithmic scale). A range of relationship between volume, and volume sum of particles corresponding to this range was performed. Three replicates were averaged in order to define particle size distribution trends.

\subsubsection{Molecular weight $\left(M_{\mathrm{w}}\right)$ distribution}

Molecular weight distribution of the PLA after processing was acquired by size exclusion chromatography (SEC). Neat-PLA, processed-PLA and powdered blends $(1 \mathrm{~g})$ were dissolved in $70 \mathrm{~mL}$ of chloroform at room temperature $\left(20-22^{\circ} \mathrm{C}\right)$ during 7 days under stirring. The resulting suspension was centrifuged for $5 \mathrm{~min}$ at $4000 \mathrm{rpm}$, and an aliquot $(50 \mu \mathrm{L})$ was analyzed using isocratic chloroformeluted at $22^{\circ} \mathrm{C}$. Instrument (GPC Shimadzu, Japan) was equipped with DAD, and RID detectors. Polystyrene-divinylbenzene standards $\left(\mathrm{Phenogel}^{\circledR}\right)$ were used for calibration.

\subsubsection{Thermal properties}

Differential scanning calorimetry (DSC)

DSC analysis was performed on a NETZSCH DSC instrument (204 F1 Phoenix, Germany). Samples $(7 \pm 3 \mathrm{mg})$ were sealed in high pressure steel pans; at a cooling rate of $10^{\circ} \mathrm{C} \cdot \mathrm{min}^{-1}$, and heated from 50 to $160^{\circ} \mathrm{C}$ at $20^{\circ} \mathrm{C} \cdot \mathrm{min}^{-1}$ heating rate under $\mathrm{N}_{2}$ atmosphere, flow rate $20 \mathrm{~mL} \cdot \mathrm{min}^{-1}$. The sample was then cooled back to $50^{\circ} \mathrm{C}$ at $10^{\circ} \mathrm{C} \cdot \mathrm{min}^{-1}$, and heated again from 50 to $250^{\circ} \mathrm{C}$ at $20^{\circ} \mathrm{C} \cdot \mathrm{min}^{-1}$. The glass transition temperature $\left(T_{\mathrm{g}}\right)$ of the blends was taken as the mid-point in the $C_{\mathrm{p}}$ step on the $2^{\text {nd }}$ run associated at the second order thermal transition.

\section{Thermal stability}

Thermal stability was carried out using thermogravimetric analysis (TGA) performed on a NETZSCH TGA instrument (TG 209 F3 Tarsus, Germany). Samples of $6 \pm 2 \mathrm{mg}$ was heated at $10^{\circ} \mathrm{C} \cdot \mathrm{min}^{-1}$ from 25 to $600^{\circ} \mathrm{C}$ under $\mathrm{N}_{2}$ atmosphere, flow rate $20 \mathrm{~mL} \cdot \mathrm{min}^{-1}$. The degradation temperature $\left(T_{\mathrm{d}}\right)$ was determined from the temperature peak obtained in the 1 st derivative curve. The $T_{5 \%}$ ( $5 \%$ of mass loss) was determined based on the TGA thermogram.

\subsection{Flexural testing}

Pressed laminated samples were cut with a die (gage length $60 \mathrm{~mm}$, width $1.5 \pm 0.2 \mathrm{~mm}$ ) and kept in a climate chamber oven overnight $\left(T: 23 \pm 1^{\circ} \mathrm{C}, p: 1.0 \mathrm{~atm}\right.$, relative humidity: $50 \pm 3 \%$ ), and the Young's modulus (E) were determined [15]. Universal tensile testing machine (SmarTens 005, KARG Industrietechnik, Germany) was used employing a crosshead speed of $10 \mathrm{~mm} \cdot \mathrm{min}^{-1}$. Eight laminate samples for each treatment were used for the flexural test analysis.

\section{Results and Discussion}

\subsection{Evaluation of processability by torque rheometer}

Selected torque-rheometer plots as a function of time at $50 \mathrm{rpm}$ is provided (Figure 2).

Modified (PRI-3), and unmodified polyflavonoids (PRI) reduced the PLA-softening point. The decreasing of the torque on PRI-based blends associated to the PLA-softening point was clear, and it can be observed around $1.5 \mathrm{~min}$. Modified polyflavonoid favors the earliest PLA-softening point $\left(t_{\text {mix }}: 0.8 \mathrm{~min}\right)$ re- 


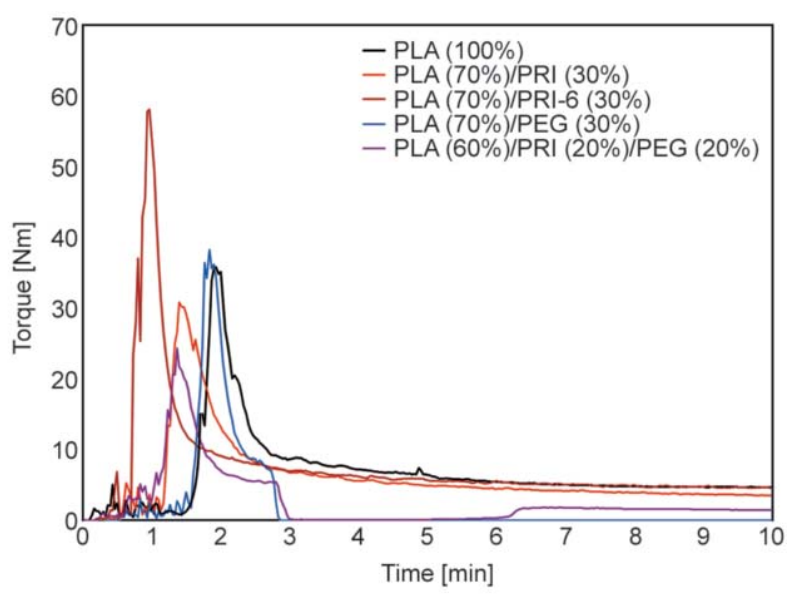

Figure 2. Rheological behaviour of neat PLA, and selected PLA-based blends prepared at mixture temperature of $170^{\circ} \mathrm{C}\left(t_{\text {mix }}: 15 \mathrm{~min}\right)$

gardless blend-composition. The dramatic influence of polyflavonoids on the PLA behavior is unexpected. However, the structure of polyphenol in terms of aromatic-content might be affecting the heat-transfer process during the mixing. In addition, the low heatcapacity of aromatic compounds in binary and ternary thermodinamical systems is a well-known issue [16]. However, the apparent low heat-capacity of polyflavonoids is not enough reason for the remarkable shifting of the PLA-softening point. Chemical interaction between PLA-chain and modified polyflavonoids grafting moieties apparently affects PLA-thermal behavior. In fact, is important to bring the homogenization times to a minimum. However, modified polyflavonoid reduced the mixing-time significantily at

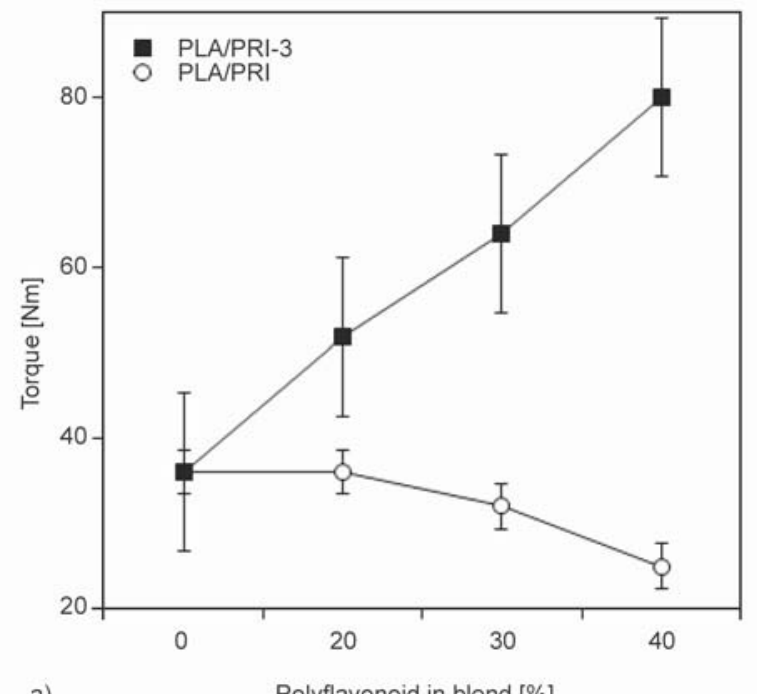

a)

Polyflavonoid in blend [\%] the expense of the higher torque values (torque: 40 $80 \mathrm{Nm}$ ). In contrast, unmodified polyflavonoids reduced slightly the torque in comparison to the neat PLA (Figure 3).

Blends prepared with modified polyflavonoid (PRI-3) induced the higher torque values. The high aliphatic $\mathrm{OH}-$ content of polyflavonoid derivatives in comparison to the lower ones of neat polyflavonoids apparently increases the viscosity during the melting, and in consequence the resistance to the rheometer blades. The differences in torque values can be explained considering that the viscoelastic properties of polyflavonoids dramatically change upon hydroxypropylation [9-11]. Is well-known that the aliphatic $-\mathrm{OH}$ functionalities enhance hydrogen-bridge interaction between polymer chains. In addition, strong association between linear aliphatic PLA's chain, and the hydroxypropyl grafting from the derivatives during blending might be also considered.

On the other hand, low interaction between neat polyflavonoids and PLA-chains can be explained in terms of the high differences in polarity of the two biopolymers $[10,17]$. However, molecular association between PLA and polyflavonoids in the molten-state has not been documented before.

\subsection{Molecular weight distribution}

A dramatic effect of the blend-composition, and the processing strategy on the PLA- $M_{\mathrm{w}}$ was observed (Figure 4).

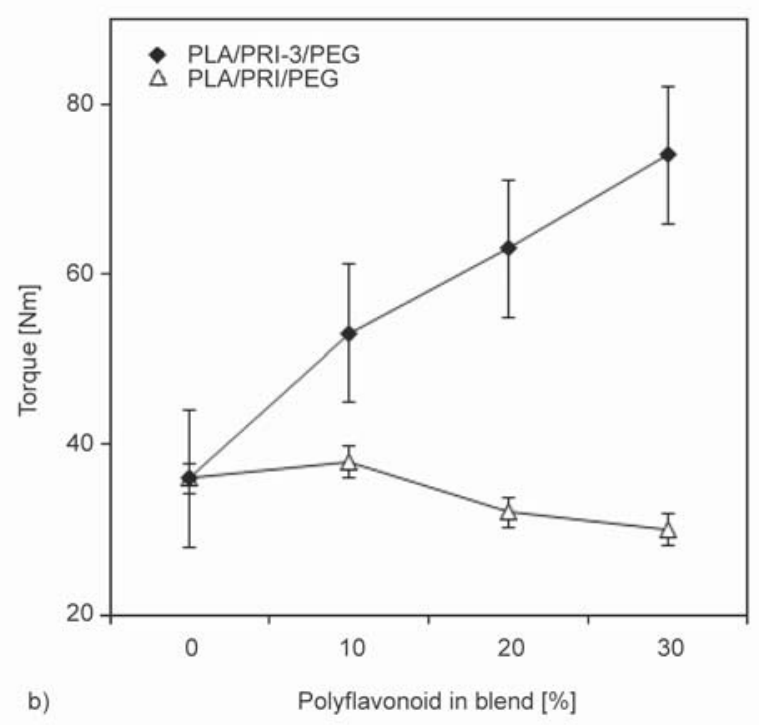

Figure 3. Maximal torque associated to the PLA-melting in function of the polyflavonoid-content for PLA-based blends prepared at $170{ }^{\circ} \mathrm{C}\left(t_{\text {mix }}: 15 \mathrm{~min}\right)$. (a) binary blends (PLA/PRI, and PLA/PRI-3). (b) ternary blends (PLA/PRI/PEG, and PLA/PRI-3/PEG). $N=3$. Note: data illustrate the torque variation during the first two minutes of mixing. 


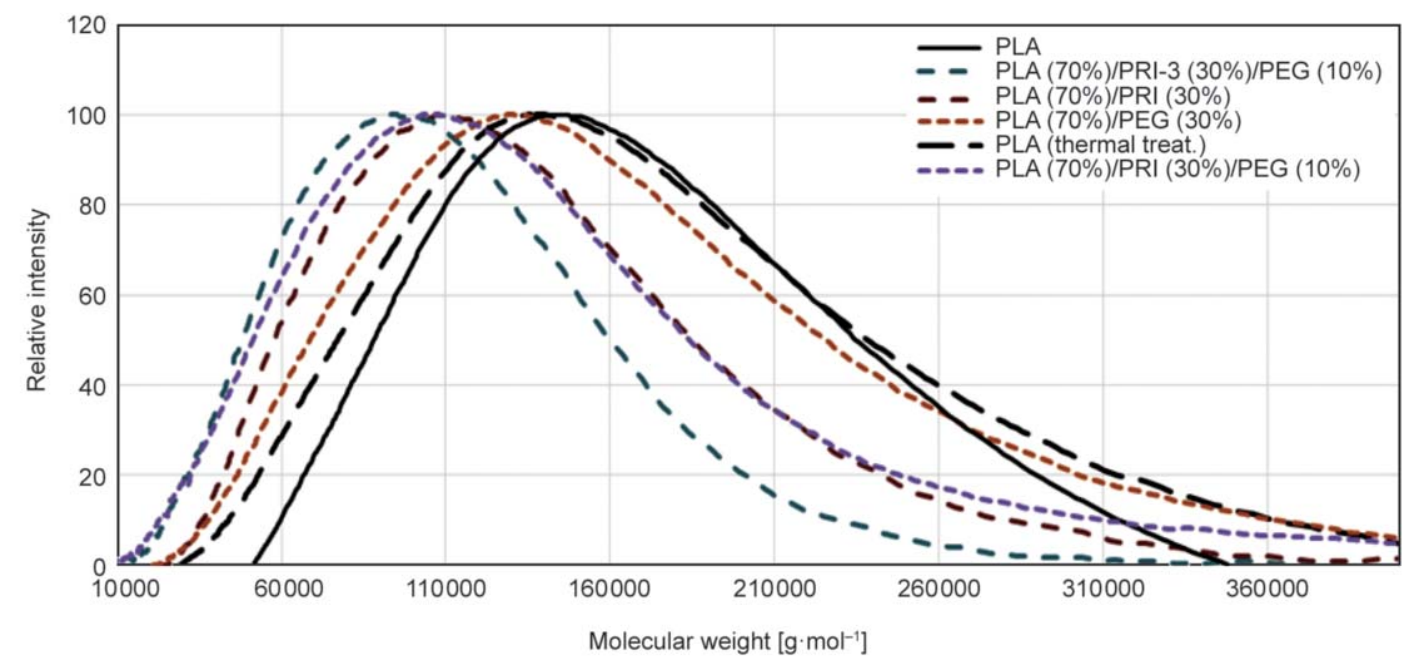

Figure 4. Selected GPC-traces of PLA from PLA-based blends illustrating changes of the molecular weight distribution. Thermal treatment: $170^{\circ} \mathrm{C}, 15 \mathrm{~min}$.

PEG as a blend-components affected sligtly the $M_{\mathrm{wPLA}}$. However, degradation of PLA in polyflavonoidbased binary blends was the highest regardless the polyflavonoid-type. The degradation mechanisms of PLA-based materials have been well-described [1, $17,18]$. In fact, several components on blends have been recognized as degradation promoters of PLAdegradation [22-26]. $M_{\mathrm{wPLA}}$ decreased in ca $40000 \mathrm{~g} \cdot \mathrm{mol}^{-1}$ suggesting random cleavages on the polymer chains apparently by the effect of the polyflavonoid acidity (natural pH: 3.0-4.5). However, 15 minutes of PLA-proccesing on the rheometer ( $T$ : $170^{\circ} \mathrm{C}$ ) also affects the $M_{\mathrm{wPLA}}$ from $15.4 \cdot 10^{4}$ to $13.8 \cdot 10^{4} \mathrm{~g} \cdot \mathrm{mol}^{-1}$.

Unmodified polyflavonoids had the biggest influence in PLA-degradation (Figure 5a). The results highlight the influence of polyphenols as the main source of PLA-degradation.

On the other hand, increased D as (dispersity) a function of the polyflavonoid-content can be interpreted as a greater diversity of degraded polymer-chains during the blending [11].

It is worth noticing that the modified polyflavonoids (PRI-3) had a minor effect on polydispersity even at the highest derivatives-charge (Figure 5b). Apparently the replacement of the acidic hydroxyl groups $(-\mathrm{OH}, p K a: 9-10)$ by the hydroxypropyl chain $(-\mathrm{OH}$, $p K a: 13-16)$ decreases the detrimental effect of polyflavonoids on PLA-degradation during processing. The remarkable influence of polyflavonoids on the $M_{\mathrm{wPLA}}$ can be used as an advantage for PLA/polyflavonoid blend formulation, considering that the
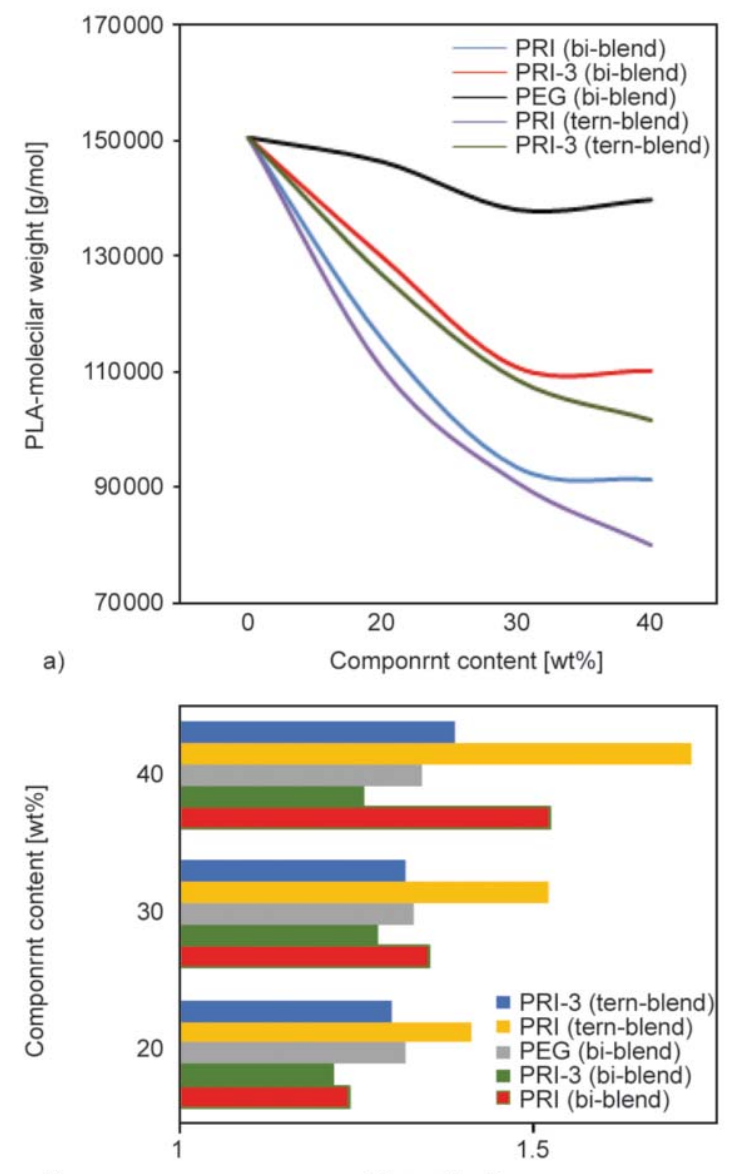

b)

Dispersity, $D$

Figure 5. Molecular weight features of PLA-based blends prepared at $170{ }^{\circ} \mathrm{C}\left(t_{\text {mix }}: 15 \mathrm{~min}\right)$. (a) Molecular weight distribution in function of the componentcontent, (b) dispersity values $(D)$ in function of the blend-type. $N=3$.

biodegradability and disintegration of PLA, and PLAbased materials are strongly associated to the $M_{\mathrm{w}}$ of 
the polymer-matrix, the crystallinity degree, and the chemical lability of selected functional groups [18]. That is why polyflavonoids can be used for tailoring PLA's molecular properties. In addition, polyflavonoids also might be advantageous as functional additives for several biopolymers such as starch, cellulose, and commercial biodegradable aliphatic copolymers $\left(\right.$ Ecoflex $^{\circledR}$ and Ecovio $\left.{ }^{\circledR}\right)[3,24,26]$.

\subsection{Morphology}

\subsubsection{Confocal fluorescence microscopy (spectrum pattern)}

Confocal fluorescence microscopy is an extremely useful tool enabling polyphenols quantification [19]. The strong fluorescence pattern of polyflavonoids can be used to estimate particle size and particle shape, considering the fact that polyflavonoid-derived composites have a typical dark appearance.

Considering that polyflavonoid blends are colored composites (image not-shown), the evaluation of fluorescence emission pattern was performed considering that PLA, and PEG are not-active fluorescent molecules. Such features were utilized in order to study the morphological features of PLA-based blends [11, 20].

The emission spectra of PRI and PRI-3 were assessed by the three types of lasers $\left(\lambda_{\mathrm{I}}: 405 \mathrm{~nm}, \lambda_{\mathrm{II}}\right.$ : $\left.488 \mathrm{~nm}, \lambda_{\text {III }}: 561 \mathrm{~nm}\right)$. Chemical modification has a negligible influence on the emission pattern (Figure 6a). Results allowed concluding that fluorescence measurements are not affected significantly by hydroxypropylation. Apparently, the structural variations upon derivatization do not affect the energy emission pattern.

Based on such findings, fluorescence emission pattern of binary and ternary PLA-based blends was assessed by two lasers $\left(\lambda_{\mathrm{I}}\right.$ and $\left.\lambda_{\mathrm{III}}\right)$ covering the entire range of wavelength. As expected, marginal differences regarding emission pattern in function of blend-composition were observed (Figure 6b).

Regardless blend-composition, maximal fluorescence emission $\left(\lambda_{\mathrm{I}}\right)$ oscillated between 480 and $520 \mathrm{~nm}$, while Laser III $(\lambda: 561 \mathrm{~nm})$ induces an emission pattern in a narrow range of wavelength (600 $630 \mathrm{~nm}$ ). That is why red light was used in order to assess the morphological features of the blends.
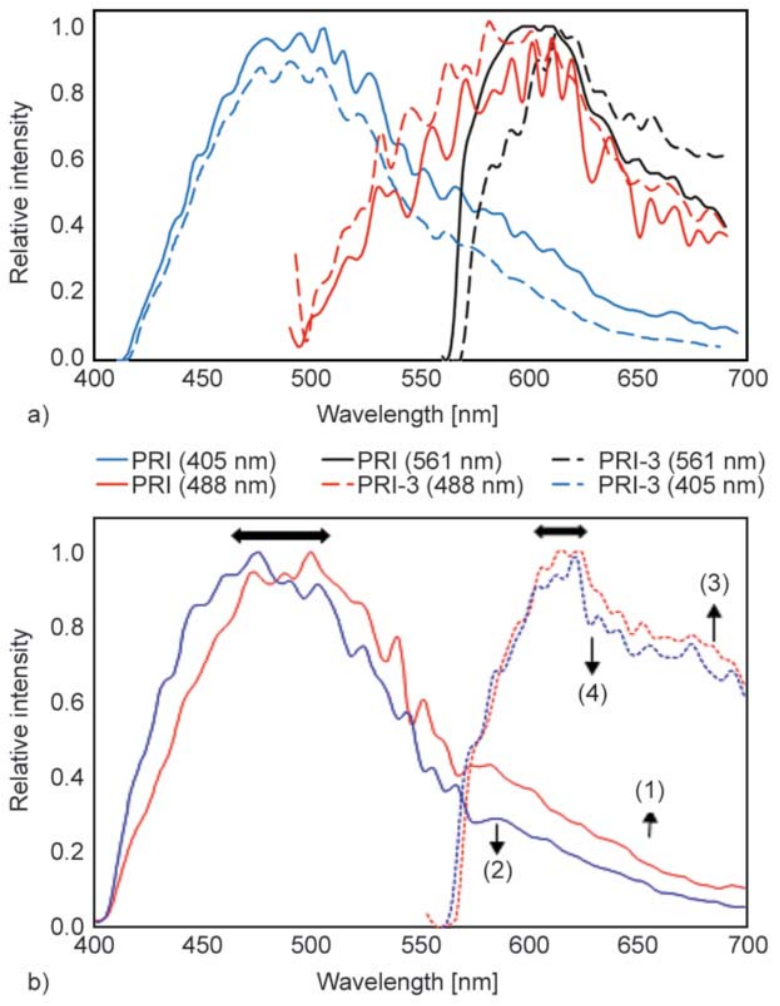

Figure 6. Fluorescense emission features of polyflavonoids, and polyflavonoids-based blends prepared at $170^{\circ} \mathrm{C}$ $\left(t_{\text {mix }}: 15 \mathrm{~min}\right)$. (a) emission spectra of modified (PRI-3), and unmodified (PRI) polyflavonoids, (b) emission spectra of PLA-based blends, (1) $\mathrm{PLA}_{70} / \mathrm{PRI}_{30}$ (Laser $\lambda_{\mathrm{I}}: 405 \mathrm{~nm}$ ), (2) $\mathrm{PLA}_{70} /$ $\mathrm{PRI}_{30}$ (Laser $\lambda_{\mathrm{II}}: 561 \mathrm{~nm}$ ), (3) $\mathrm{PLA}_{60} / \mathrm{PRI}-3_{20} /$ $\mathrm{PEG}_{20}$ (Laser $\lambda_{\mathrm{I}}: 405 \mathrm{~nm}$ ), and (4) $\mathrm{PLA}_{60} / \mathrm{PRI}-3_{20} /$ $\mathrm{PEG}_{20}$ (Laser $\lambda_{\mathrm{II}}: 561 \mathrm{~nm}$ ).

\subsubsection{Particle size distribution (image-processing)}

Maximal intensity projection of selected PLA-based blends is shown (Figure 7).

Image-processing revealed remarkable differences in morphology of polyflavonoid particles dispersed on the PLA-matrix $(N)$. The methodology allowed a high count of particles. Particle volumes were determined on the basis of a selective channel (red, $\lambda_{\text {III: }}$ : $561 \mathrm{~nm}$ ). PRI-3-based blends exhibited the smallest particles volume, which describes the better miscibility of polyflavonoid derivatives (Figure 8a), whereas the particle size of PRI, and PRI-3 were rather similar (see Figure 8b).

However, PRI particles volume was the biggest in ternary blends. Apparently, certain interaction between polyflavonoids and PEG affects significantly the con- 


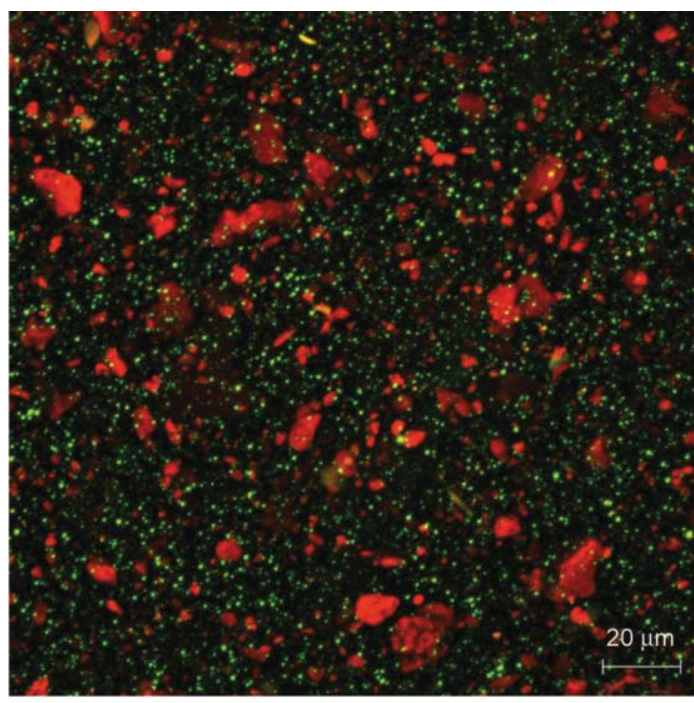

a)

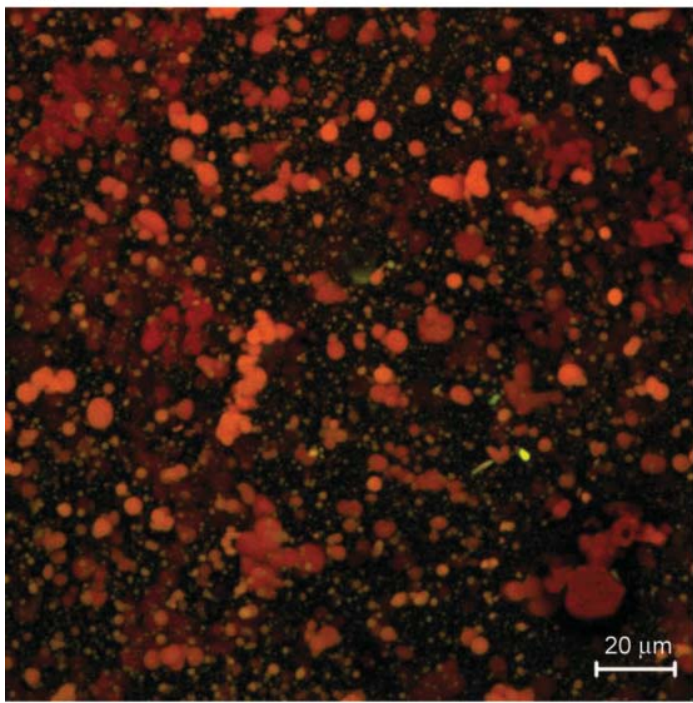

c)

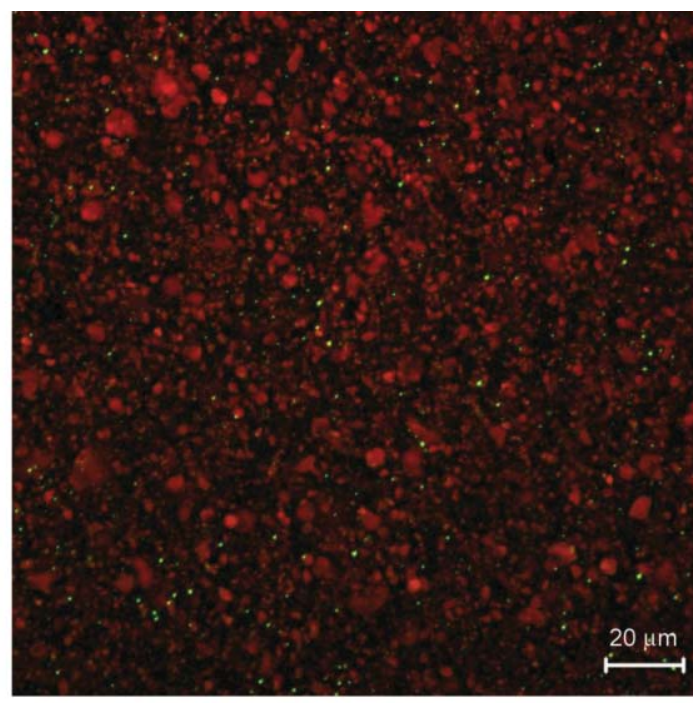

b)

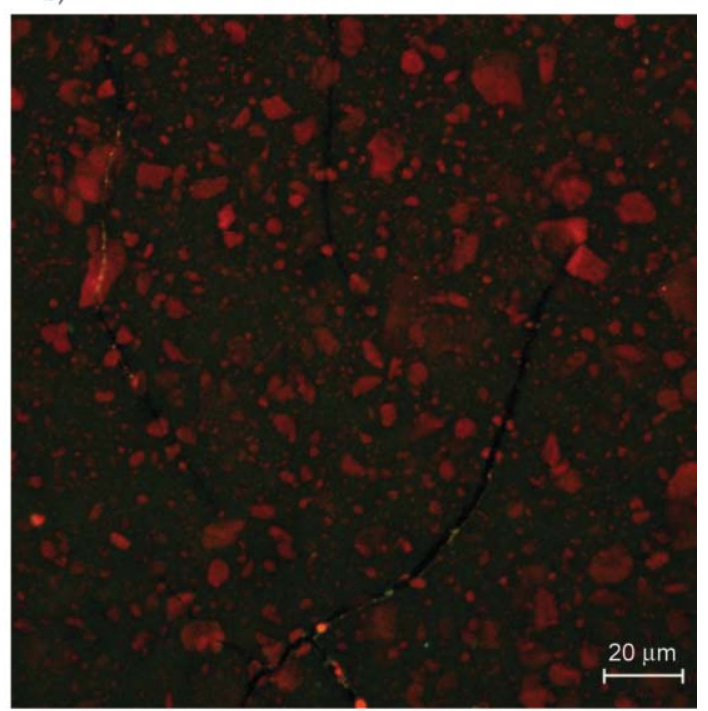

d)

Figure 7. Maximum intensity projection of selected PLA-based blends prepared at $170{ }^{\circ} \mathrm{C}\left(t_{\text {mix }}: 15\right.$ min). (a) $\mathrm{PLA}_{70} / \mathrm{PRI}_{30}$, (b) $\mathrm{PLA}_{70} / \mathrm{PRI}-3_{30}$, (c) $\mathrm{PLA}_{60} / \mathrm{PRI}_{20} / \mathrm{PEG}_{20}$, (d) $\mathrm{PLA}_{60} / \mathrm{PRI}-3_{20} / \mathrm{PEG}_{20}$.

formational features during the blending. The result matches with the spherical particle morphology clearly visible on the maximum intensity projection image (see Figure 7c). In fact, is well-known that PEG interacts strongly with polyflavonoids via hydrophobic domains $[11,21]$. The $\mathrm{H}-\mathrm{H}$ interaction enables an open-conformation of the polyflavonoid molecules, so increasing volumen was apparently associated to molecular complexation during the melt-blending. Normalized histogram (Figure 8) shows that polyflavonoid chemical modification enhances the miscibility on the PLA-matrix regardless the blend-type. On the other hand, unmodified polyflavonoids show similar particle volume distribution to the referential PRI volume (see Figure 8b). Limited solubility of polyphenols on the PLA-matrix can be explained considering the high difference in polarity between PRI, and PLA.

As described previously by image-processing, PEG affects dramatically the polyflavonoid particle volume.

The low polarity of PRI-3 is a key factor to understand the better compatibility features of modified polyflavonoids on the PLA-matrix. Maximum interaction between the hydroxypropyl moieties with the PLAchain, and PEG during the mixing highlights that the chemical modification is a feasible strategy in using polyflavonoids in bioplastics.

In addition, it worth to highlight that confocal microscopy (fluorescense emission) coupling to image- 


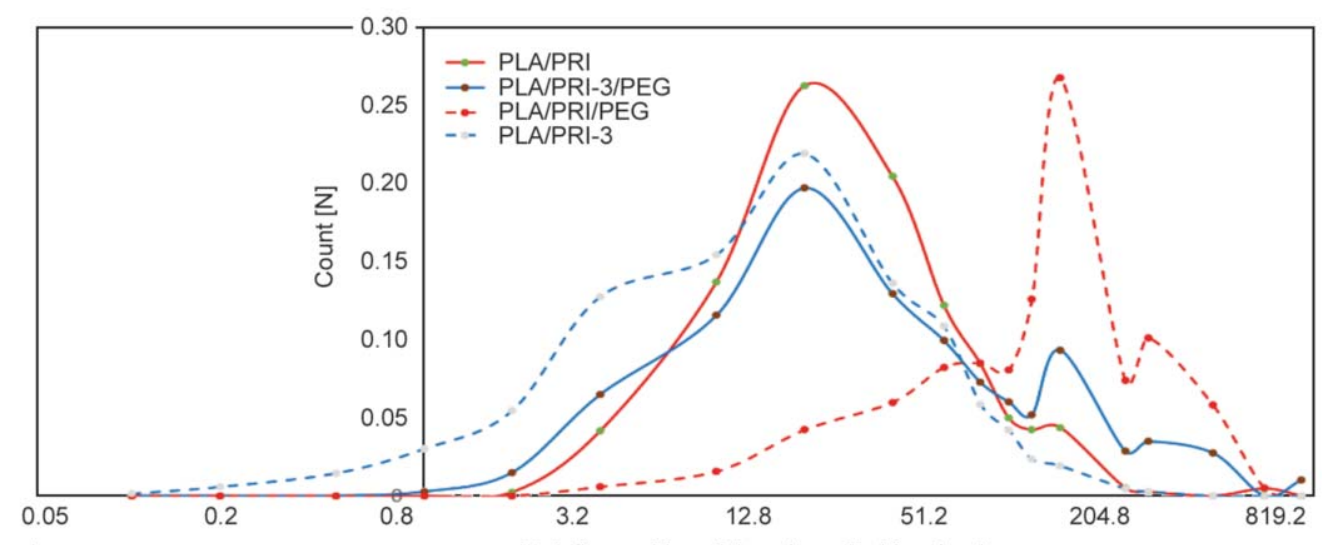

a)

Polyflavonoid particle volume [cubic micra]

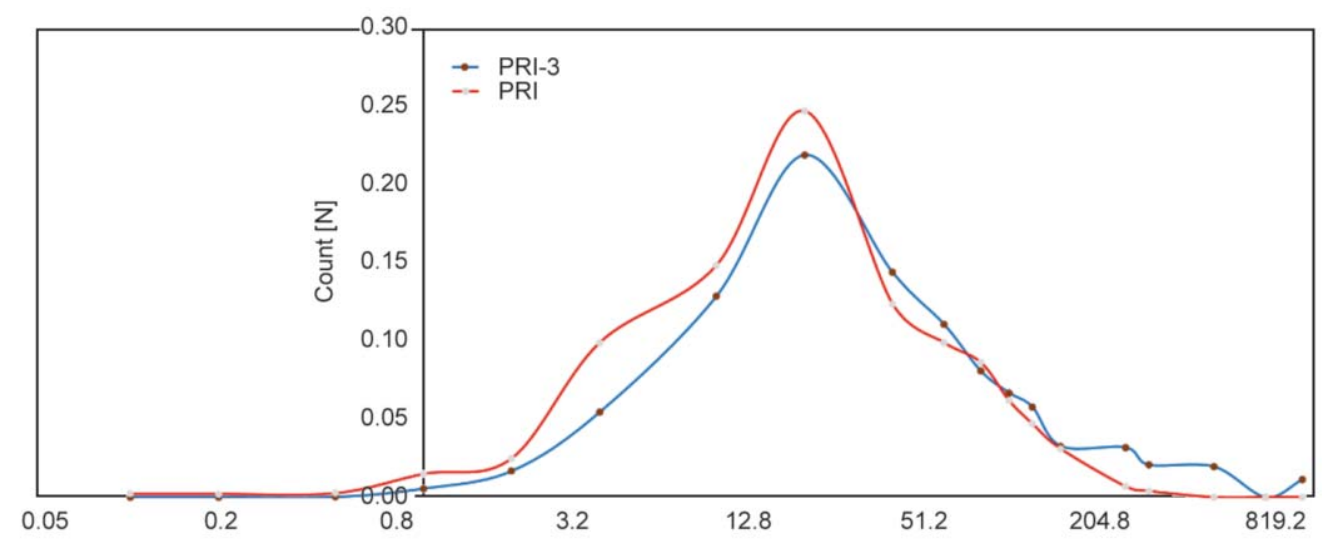

b)

Polyflavonoid particle volume [cubic micra]

Figure 8. Normalized polyflavonoids particle volume of PLA-based blends assessed by emission fluorescence microscopy. (a) Polyflavonoids particle volumen (reference), (b) polyflavonoids particle volumen in blends (binary blend: $\mathrm{PLA}_{70}$, ternary blends: $\mathrm{PLA}_{60} / \mathrm{PEG}_{10 \%}$ ). Note: polyflavonoid particles volume is expressed in logarithmic scale.

processing is a usefull technique in estimating the morphological parameters of PLA biopolymer blended with natural polyflavonoids.

\subsection{Thermal properties}

\subsubsection{DSC analysis}

Selected thermograms illustrate the $T_{\mathrm{g}}$, and $T_{\mathrm{m}}$ of the neat PLA, and PLA-based blends (Figure 9). It is evident that neat PLA has a $T_{\mathrm{g}}$ of $60^{\circ} \mathrm{C}$, and $T_{\mathrm{m}}$ of $170^{\circ} \mathrm{C}$. In binary blends polyflavonoids did not significantly influence the $T_{\mathrm{g} \text { PLA }}$ value. However, such polyphenols induce the PLA-crystallization $\left(T_{\mathrm{c}}\right.$ : $100^{\circ} \mathrm{C}$ ) to a high extent. The nucleating effect of polyflavonoids in PLA-based blends has been not wellclarified in previous works. Differences in polarity, hydroxyl-content, and conformational features are factors apparently affecting the PLA-crystallization behavior induced by polyflavonoids.

On the other hand, PEG induces a reduction of $T_{\mathrm{gPLA}}$ $\left(55.6^{\circ} \mathrm{C}\right)$, as well as the $T_{\mathrm{CPLA}}$. The synergetic effect of PEG on the PLA-thermal properties has been pre- viously reported, as well as on the crystallization behavior [22-26]. The remarkable effect on thermal properties can be explained considering that $\mathrm{PEG}$ favors the breaking of intermolecular hydrogen bridges of PLA during processing, prone to an early melting, and a significant $T_{\mathrm{g}}$ reduction.

In addition, Figure $9 b, 9 c$ shows in detail the thermal behavior of all tested blends according to the component-loading. Polyflavonoid induces a significant $T_{\text {mPLA }}$ and $T_{\text {cPLA }}$ reduction in ternary blends. However, the variation of $T_{\mathrm{g}}, T_{\mathrm{c}}$, and $T_{\mathrm{m}}$ in binary blends was negligible. In contrast, PEG significantly affects the $T_{\mathrm{gPLA}}, T_{\mathrm{mPLA}}$ and $T_{\mathrm{cPLA}}$ values.

It is worth noticing that the depression of the $T_{\mathrm{gPLA}}$, and $T_{\mathrm{cPLA}}$ was remarkable in PRI-3-based blends. The results describe the advantageous effect of the chemical modification on the miscibility features.

Apparently, polyflavonoids act as an effective nucleating agent in PLA-based blends. The PLA-crystallization during the blend-cooling has an important impact on the mechanical properties. Several salts 

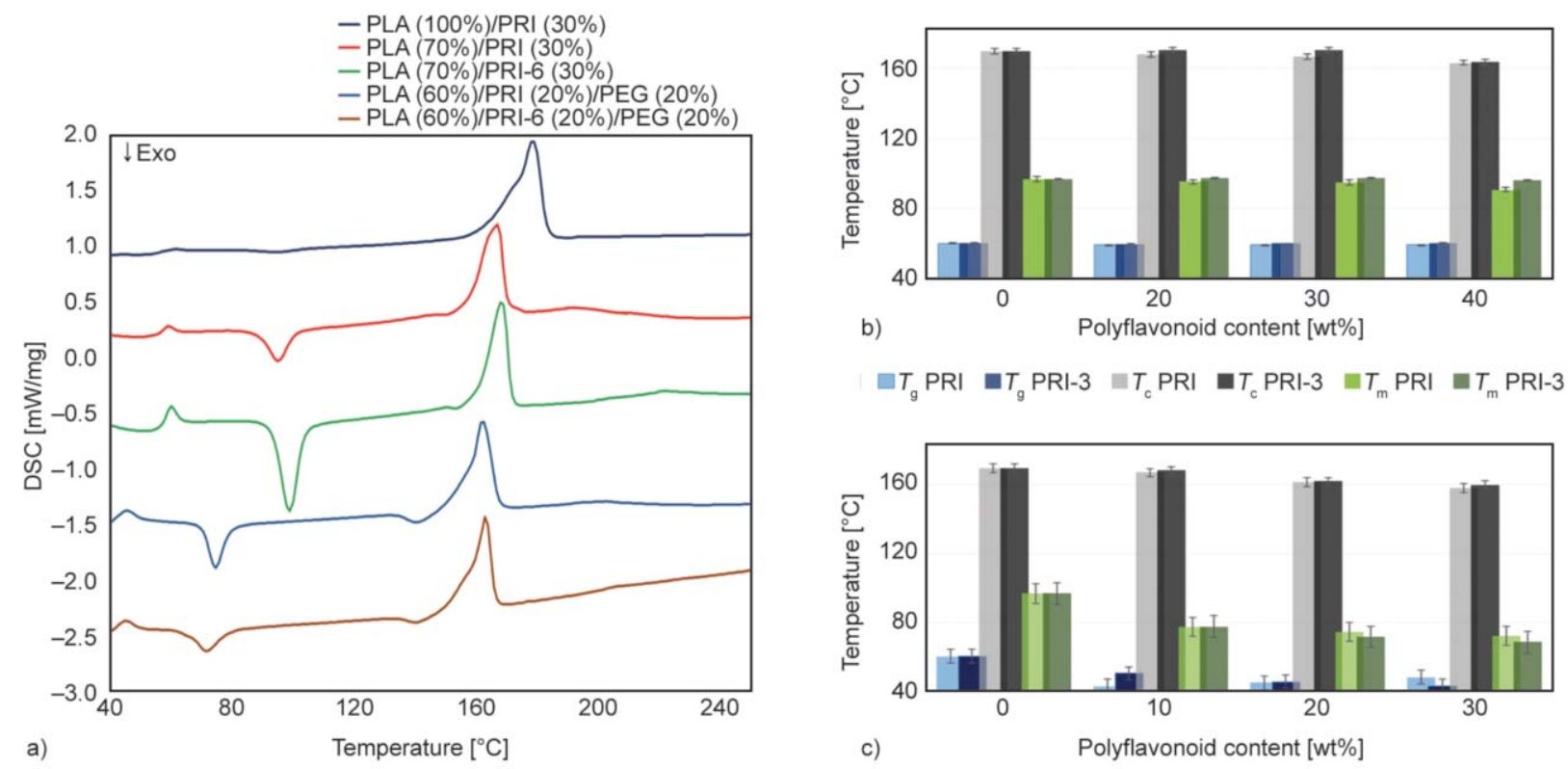

Figure 9. Thermal behavior (DSC) of PLA-based blends prepared at $170^{\circ} \mathrm{C}\left(t_{\text {mix }}: 15 \mathrm{~min}\right)$ considering the second heating. (a) Selected DSC-traces, (b) transitional temperatures in function of polyflavonoid-content (binary blends), (c) transitional temperatures in function of polyflavonoid-content (ternary blends). $N=3$.

(talcs), organic compounds (amines), fibers, nanotubes, and commercial nucleating (3801X, and LAK) have been reported as nucleating agent in PLA-based blends $[23,24]$. However, to our knowledge it is the first time that polyphenols from pine bark are reported as nucleating agents in PLA-matrix in a wide range of composition.

The synergic effect between PEG and polyflavonoids on the PLA thermal behavior $\left(T_{\mathrm{g}}, T_{\mathrm{c}}, T_{\mathrm{m}}\right)$ has been also reported in several systems $[25,26]$. The similarities regarding functional groups of PEG and PLA apparently favor the mobility of the chain and breaks the intermolecular hydrogen bonds which reduce transitional temperatures.

Since the $T_{\mathrm{g}}$ value is an excellent indicator of chain mobility, plasticizing efficiency has therefore been evaluated by measuring the $T_{\mathrm{g}}$ shifting as a function of plasticizer concentration. All the binary blends show only one $T_{\mathrm{g}}$ in the DSC-thermogram indicating an apparent good miscibility between PLA, PRI-3, and PEG. The decrease of $T_{\mathrm{g}}$ highlights the effect of the plasticizer in PLA compositions with increasing molecular mobility. The plasticizing effect is confirmed by the modification of $T_{\mathrm{g}}$ which is significantly decreased by comparison to the neat PLA ma$\operatorname{trix}\left(T_{\mathrm{g}}: \sim 60^{\circ} \mathrm{C}\right)$.

\subsubsection{TGA analysis}

The selected thermograms showed that a significant change to the degradation temperatures according to the blend-composition was observed (Figure 10). The $T_{\mathrm{d}}$ values were above $300^{\circ} \mathrm{C}$. However, a remarkable effect of components on the $T_{5 \%}$, and $T_{\mathrm{d}}$ of ternary blends was noticed.

Thermal behavior of binary and ternary-blends reveals a significant effect of the polyflavonoids-content (Figure 10).

Neat polymers (PLA, PEG), exhibited a single decomposition peak, indicating that they degraded mainly in one step (data not-shown). Polyflavonoidcontent affects the $T_{5 \%}$, and $T_{\mathrm{d}}$ significantly. The results can be explained considering that polyphenols are thermally unstable building-blocks, prone to yield carbonaceous residues as a consequence of pyrolytic processes $[9,11]$.

PRI-3-based ternary blends show the lowest $T_{\mathrm{d}}$. The result is unexpected considering the high thermal stability of PRI-3 in comparison to unmodified bark polyflavonoids described in previous reports [11]. However, polyflavonoid's DS cannot be considered as the unique factor affecting thermal decomposition of the blends. The substitution pattern on the $\mathrm{C}_{15}$ polyflavonoid unit (see Figure 1) seems to be a key factor 

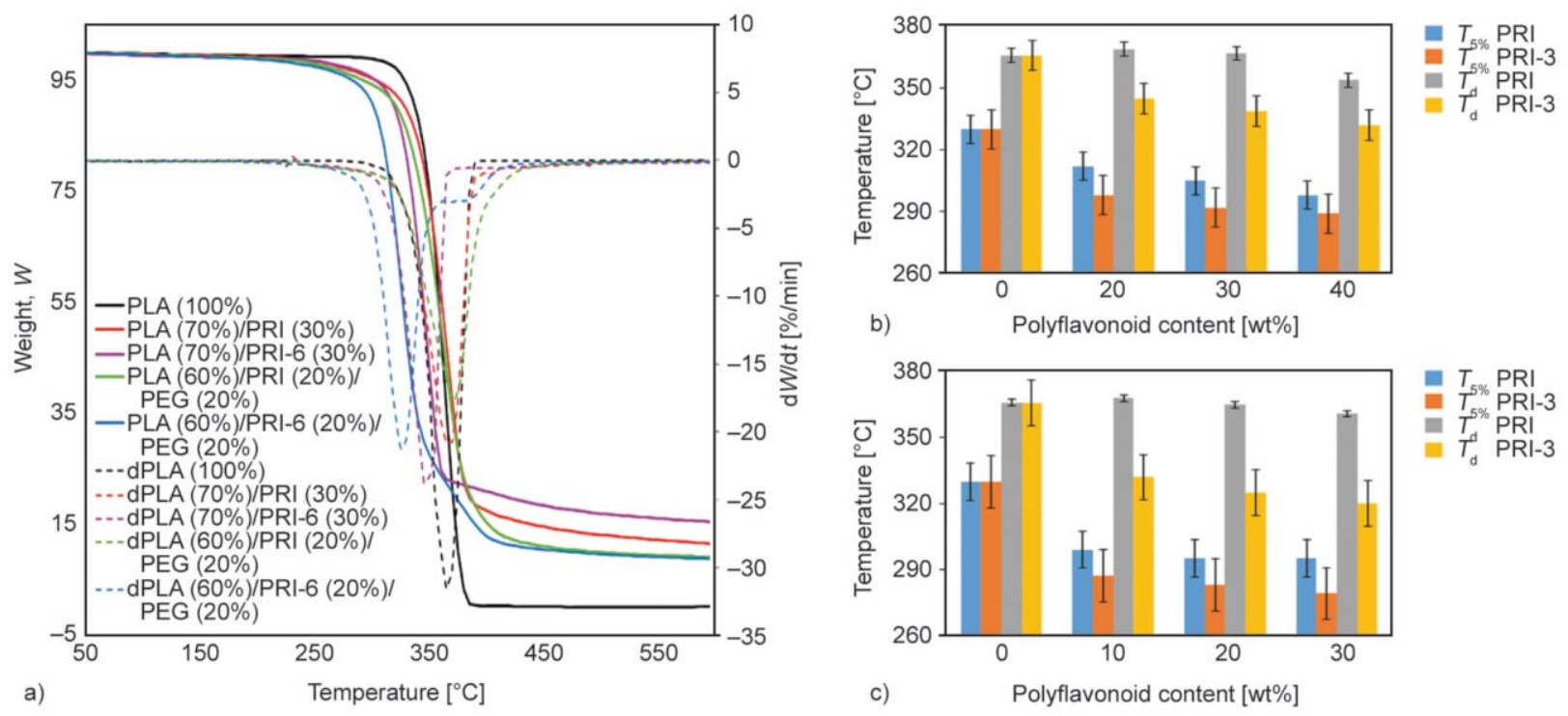

Figure 10. Thermal behavior (TGA) of PLA-based blends prepared at $170^{\circ} \mathrm{C}\left(t_{\text {mix }}: 15 \mathrm{~min}\right)$. (a) Selected TGA-traces, (b) decomposition temperatures in function of polyflavonoid-content (binary blends), (c) decomposition temperatures in function of polyflavonoid-content (ternary blends). $N=3$.

for polyphenols degradation $[8,9]$. The low $M_{\mathrm{w}}$ distribution of the water-insoluble tannin fraction (PRI), as well as the lipophilic character of such polyflavonoids as a consequence of concomitants apparently affects the thermal stability of the blends in a differentiated manner.

On the other hand, all PLA-blends showed significant residues at $600{ }^{\circ} \mathrm{C}(<20 \%)$. Residual weight increased with increasing polyflavonoid-content, excepting in PLA-based ternary blends (Figure 11). The results point out that the polyflavonoid-based pyrolytic residues are significant products during the blend decomposition. However, a strong interaction between PEG, and polyflavonoids on the residual

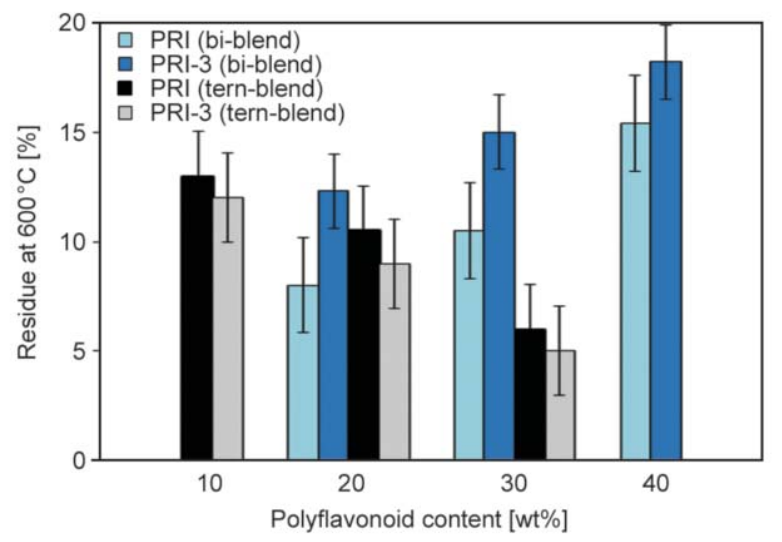

Figure 11. Residual weight at $600^{\circ} \mathrm{C}$ of binary-, and ternaryblends prepared at $170^{\circ} \mathrm{C}\left(t_{\text {mix }}: 15 \mathrm{~min}\right) . N=3$. weight was evidenced. Apparently, polyphenol-PEG complexation favored the volatilization rate at high temperatures $\left(>400^{\circ} \mathrm{C}\right)$.

\subsection{Flexural testing}

PLA-based blends exhibited a typical stress-strain curve which describes that the samples breaks before yielding (Figure 12). Curve morphology was affected by the blend-composition.

The flexural behaviour of the neat PLA showed characteristics of glassy polymers with low deformation at break. In contrast, PLA-based blends showed a different behaviour (Table 2).

In general, PLA/PRI blends show poor performance in term of Young's modulus $(E)$. In contrast, the highest PRI-3-charge the highest $E$-values. The behavior can be explained by incompatibility between the neat polyflavonoids, and the PLA polymer due to the difference in polarity, and molecular features.

In addition, the $E$-modulus value dropped dramatically from 88 to $22 \mathrm{MPa}$ with incorporation of PRI (10 wt\%); indicating incompatibility. In contrast, Emodulus was improved in $15 \mathrm{MPa}$ when PRI-3 was loaded from 10 to $30 \mathrm{wt} \%$. This can be explained by the role of hydroxypropyl-chain which apparently acts as a compatibilizer moiety in binary-, and ternary-blends. 

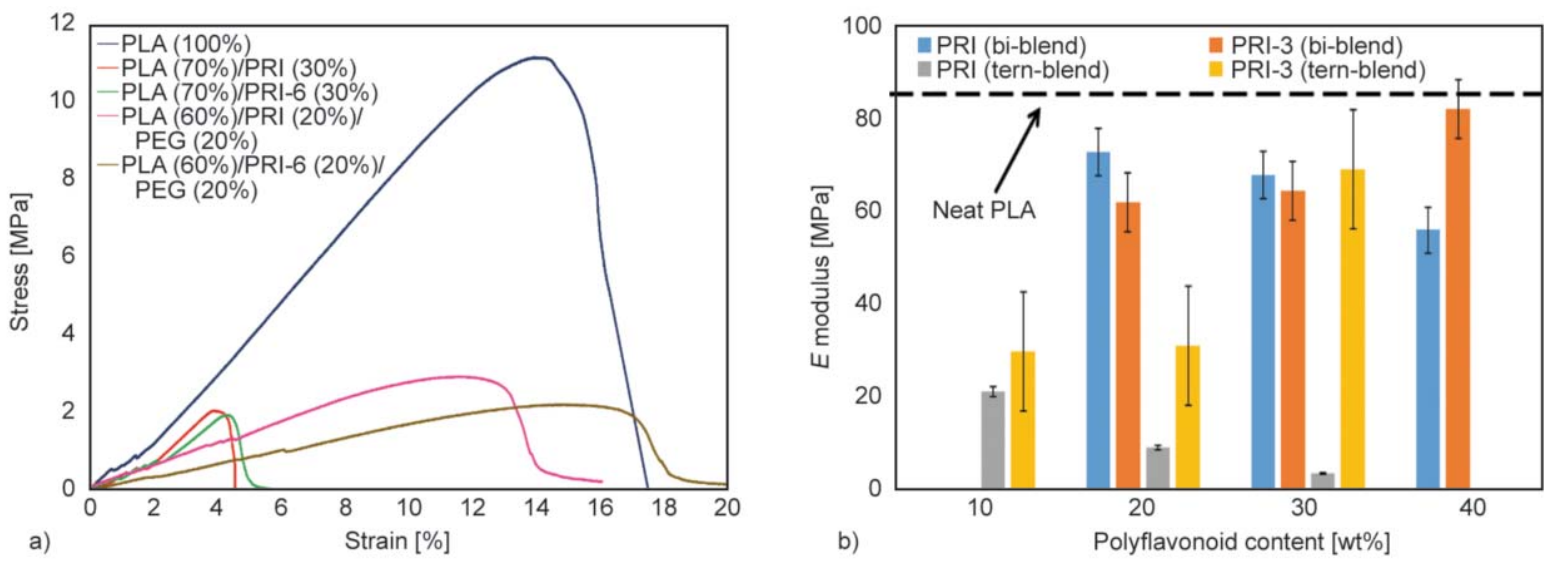

Figure 12. Mechanical performance of PLA-based blends prepared at $170{ }^{\circ} \mathrm{C}\left(t_{\text {mix }}: 15 \mathrm{~min}\right)$. (a) Selected stress-strain curves, (b) $E$-modulus in function of the polyflavonoid content. $N=8$.

Table 2. Mechanical performance of PLA-based blends prepared at $170^{\circ} \mathrm{C}$ for $15 \mathrm{~min}$

\begin{tabular}{|c|c|c|c|}
\hline Blend & $\begin{array}{l}F_{\mathbf{m}} \\
{[\mathbf{N}]} \\
\end{array}$ & $\begin{array}{c}\sigma_{\mathrm{fM}} \\
{[\mathrm{MPa}]}\end{array}$ & $\begin{array}{c}\varepsilon_{\mathrm{fM}} \\
{[\%]}\end{array}$ \\
\hline PLA & $37.5 \pm 5$ & $2.70 \pm 1.0$ & $16.6 \pm 5.0$ \\
\hline $\mathrm{PLA}_{80} / \mathrm{PRI}_{20}$ & $18.1 \pm 3$ & $0.30 \pm 0.01$ & $6.1 \pm 0.1$ \\
\hline $\mathrm{PLA}_{70} / \mathrm{PRI}_{30}$ & $12.4 \pm 2$ & $0.15 \pm 0.01$ & $4.5 \pm 0.3$ \\
\hline $\mathrm{PLA}_{60} / \mathrm{PRI}_{40}$ & $7.2 \pm 1$ & $0.11 \pm 0.01$ & $2.12 \pm 0.1$ \\
\hline $\mathrm{PLA}_{80} / \mathrm{PRI}-3_{20}$ & $13.6 \pm 5$ & $1.82 \pm 0.21$ & $4.13 \pm 0.5$ \\
\hline $\mathrm{PLA}_{70} / \mathrm{PRI}-3_{30}$ & $10.0 \pm 2$ & $1.88 \pm 0.30$ & $4.23 \pm 0.4$ \\
\hline $\mathrm{PLA}_{60} / \mathrm{PRI} 3_{40}$ & $2.0 \pm 1$ & $1.62 \pm 0.41$ & $1.12 \pm 0.1$ \\
\hline $\mathrm{PLA}_{60} / \mathrm{PRI}_{10} / \mathrm{PEG}_{30}$ & $11.3 \pm 2$ & $0.46 \pm 0.33$ & $14.7 \pm 0.9$ \\
\hline $\mathrm{PLA}_{60} / \mathrm{PRI}_{20} / \mathrm{PEG}_{20}$ & $10.6 \pm 2$ & $0.19 \pm 0.02$ & $12.8 \pm 0.9$ \\
\hline $\mathrm{PLA}_{60} / \mathrm{PRI}_{30} / \mathrm{PEG}_{10}$ & $9.6 \pm 1$ & $0.09 \pm 0.01$ & $11.6 \pm 0.7$ \\
\hline $\mathrm{PLA}_{60} / \mathrm{PRI}-3_{10} / \mathrm{PEG}_{30}$ & $8.0 \pm 1$ & $0.21 \pm 0.02$ & $12.4 \pm 0.9$ \\
\hline $\mathrm{PLA}_{60} / \mathrm{PRI} 3_{20} / \mathrm{PEG}_{20}$ & $10.4 \pm 2$ & $0.25 \pm 0.02$ & $10.87 \pm 0.6$ \\
\hline $\mathrm{PLA}_{60} / \mathrm{PRI}_{3} 3_{30} / \mathrm{PEG}_{10}$ & $15.0 \pm 3$ & $0.46 \pm 0.03$ & $14.4 \pm 0.3$ \\
\hline
\end{tabular}

Average \pm standard deviation, $F_{\mathrm{m}}$ : maximum force, $\sigma_{\mathrm{fM}}$ : flexural strength, $\varepsilon_{\mathrm{fM}}$ : flexural strain. $N=8$.

\section{Conclusions}

PLA-based blends have been successfully prepared with $P$. radiata bark polyflavonoids, by melt-blending at $170^{\circ} \mathrm{C}$ during $15 \mathrm{~min}$. This compounding strategy is easy and potentially commercially viable for PLA applications beyond the food-packaging.

Modification of radiata pine polyflavonoids is a valuable alternative in order to improve the performance of such building-blocks in binary-, and ternary-blends. Chemical structure of the polyflavonoid's grafting influences: (1) the blend processability, (2) the component miscibility, (3) the PLA's melting-, and crystallization-processes, (4) the thermal resistance, and (5) the flexural performance.

Rheological behavior of PLA-based blends is dramatically affected by the type-, and component-compo- sition. Considering the torque-rheometer data modified, and unmodified radiata pine polyflavonoids improved the processability of PLA in term of a shortmixing.

The image-processing using confocal fluorescence microscopy was successfully utilized in order to estimate the particle volume as an indicator of polyflavonoid's miscibility. The modified polyphenols showed higher compatibility features as a consequence of the dramatic reduction of the particle volume.

Polyflavonoid induces PLA-crystallization in a high extent. The synergetic effect of PEG with polyflavonoids is related to the chemical similarity of the polymer chains enhanced upon grafting (hydroxypropyl-chain). The result is highly relevant in view of polyflavonoid-based composites preparation.

Modified polyphenol decreases slightly the thermal stability of the blends below $300^{\circ} \mathrm{C}$. Such drawback is negligible considering that the PLA-processing temperature is around $170^{\circ} \mathrm{C}$.

On the other hand, the tannin acidity affects the PLA molecular weight distribution which influences the flexural performance. Also the thermal processing of the neat PLA affects the $M_{\mathrm{w}}$ of the polymer.

The increasing of the $E$-modulus of the blends as effect of a good mixing of polyflavonoids derivatives on the PLA-matrix was achieved.

$P$. radiata bark polyflavonoids might be used for PLA-based blends formulations in view of novel envisaged applications. Polyflavonoid-based composites seem to play a key role in polymer engineering in a near future. 


\section{Acknowledgements}

D.E. García thanks to the International PhD. Student Exchange Program from Technological Development Unit (UDT), Concepción University, Chile (Basal Project: PFB27). D.E. García would like to acknowledge to the 'Comisión Nacional de Investigación Científica y Tecnológica CONICYT, Chile' (FONDECYT Project: 11150056) for the financial support. We would also like to show our gratitude to Mrs. Corina Silva, Mrs. Carmen Pradenas, and Mrs. Yohana Sanzana, for their technical assistance. In addition, authors also like to thanks to MSc. Joanna Tereszczuk, and Germán Osorio (Advance Microscopy Center, CMA), from Concepción University, Chile, for the technical assistance regarding confocal microscopy, and image-processing, respectively.

\section{References}

[1] James L.: Large-scale production, properties and commercial applications of polylactic acid polymers. Polymer Degradation and Stability, 59, 145-152 (1998). DOI: 10.1016/S0141-3910(97)00148-1

[2] Cartier L., Okihara T., Ikada Y., Tsuji H., Puiggali J., Lotz B.: Epitaxial crystallization and crystalline polymorphism of polylactides. Polymer, 41, 8909-8919 (2000). DOI: $10.1016 / \mathrm{S} 0032-3861(00) 00234-2$

[3] Frone A. N., Berlioz S., Chailan J-F., Panaitescu D. M.: Morphology and thermal properties of PLA-cellulose nanofibers composites. Carbohydrate Polymers, 91, 377-384 (2003).

DOI: $10.1016 /$ j.carbpol.2012.08.054

[4] Grigsby W. J., Kadla J. F.: Evaluating poly(lactic acid) fiber reinforcement with modified tannins. Macromolecular Materials and Engineering, 299, 368-378 (2014). DOI: $10.1002 /$ mame.201300174

[5] Eisenberg P.: Biocomposites from microfibrillated cellulose and biodegradable polymers (PLA) (in Spanish). in ' $3{ }^{\text {er }}$ Congreso Iberoamericano sobre Biorrefinerías (CIAB), $4{ }^{\text {to }}$ Congreso Latinoamericano sobre Biorrefinerías y $2^{\text {do }}$ Simposio Internacional sobre Materiales Lignocelulósicos, Concepción, Chile' 108-109 (2015).

[6] Case F. H., Honeycutt J. D.: Will my polymer mix? Methods for studying polymer miscibility. Trends in Polymer Science, 2, 256-267 (1994).

[7] Anwer M. A. S., Naguib H. E., Celzard A., Fierro V.: Comparison of the thermal, dynamic mechanical and morphological properties of PLA-lignin \& PLA-polyflavonoid particulate green composites. Composites Part B: Engineering, 82, 92-99 (2015).

DOI: $10.1016 /$ j.compositesb.2015.08.028

[8] García D. E., Glasser W. G., Pizzi A., Lacoste C, Laborie M-P.: Polyphenolic resins prepared with maritime pine bark tannin and bulky-aldehydes. Industrial Crops and Products, 62, 84-93 (2014). DOI: $10.1016 / \mathrm{j}$. indcrop.2014.08.010
[9] García D. E., Glasser W. G., Pizzi T. A., Osorio-Madrazo A., Laborie M-P.: Synthesis and physicochemical properties of hydroxypropyl tannins from maritime pine bark (Pinus pinaster Ait.). Holzforschung, 68, 411-418 (2014).

DOI: $10.1515 / \mathrm{hf}-2013-0145$

[10] García D. E., Glasser W. G., Pizzi A., Paczkowski S., Laborie M-P.: Hydroxypropyl tannin from Pinus pinaster bark as polyol source in urethane chemistry. European Polymer Journal, 67, 152-165 (2015). DOI: $10.1016 /$ j.eurpolymj.2015.03.039

[11] García D. E.: Pinus pinaster (Ait.) bark polyflavonoid and its hydroxypropyl derivatives as building-blocks for bio-material design. PhD Thesis. Freiburg University (2014).

[12] Bocalandro C., Sanhueza V., Gómez-Caravaca A. M., González-Álvarez J., Fernández K., Roeckel M., Rodríguez-Estrada M. T.: Comparison of the composition of Pinus radiata bark extracts obtained at bench- and pilot-scales. Industrial Crops and Products, 38, 21-26 (2012).

DOI: $10.1016 /$ j.indcrop.2012.01.001

[13] Jerez M., Selga A., Sineiro J., Torres J. L., Núñez M. J.: A comparison between bark extracts from Pinus pinaster and Pinus radiata: Antioxidant activity and procyanidin composition. Food Chemistry, 100, 439444 (2007). DOI: $10.1016 /$ j.foodchem.2005.09.064

[14] Pizzi A.: Polyflavonoids: Major sources, properties and applications. in 'Monomers, polymers and composites from renewable resources' (eds.: Belgacem N., Gandini A.) Elsevier, Amsterdam 179-199 (2008).

[15] ISO 527-1 5B: Plastics - Determination of tensile properties - Part 2: Test conditions for moulding and extrusion plastics (2012).

[16] Rihani D. N., Doraiswamy L. K.: Estimation of heat capacity of organic compounds from group contributions. Industrial and Engineering Chemistry Fundamentals, 4, 17-21 (1965). DOI: $10.1021 / \mathrm{i} 160013 \mathrm{a} 003$

[17] Vert M., Schwarch G., Coudane J.: Present and future of PLA polymers. Journal of Macromolecular Science Part A: Pure and Applied Chemistry, 32, 787-796 (1995). DOI: $10.1080 / 10601329508010289$

[18] Tokiwa Y., Calabia B. P., Ugwu U., Aiba S.: Biodegradability of plastics. International Journal of Molecular Sciences, 10, 3722-3742 (2009). DOI: $10.3390 /$ ijms 10093722

[19] Scogings P., Siko S., Taylor R.: Calibration of a handheld instrument for measuring condensed tannin concentration based on UV- and red-excited fluorescence. African Journal of Range and Forage Science, 31, 5558 (2014). DOI: $10.2989 / 10220119.2013 .858772$ 
[20] Grigsby W. J., Bridson J. H., Lomas C., Elliot J-A.: Esterification of condensed tannins and their impact on the properties of poly(lactic acid). Polymers, 5, 344360 (2013).

DOI: $10.3390 /$ polym5020344

[21] Makkar H. P. S., Blümmel M., Becker K.: Formation of complexes between polyvinyl pyrrolidones or polyethylene glycols and tannins, and their implication in gas production and true digestibility in in vitro techniques. British Journal of Nutrition, 73, 897-913 (1995).

DOI: $10.1079 /$ BJN19950095

[22] Shi X., Zhang G., Vu Phuong T., Lazzeri A.: Synergistic effects of nucleating agents and plasticizers on the crystallization behavior of poly(lactic acid). Molecules, 20, 1579-1593 (2015).

DOI: $10.3390 /$ molecules20011579
[23] Li H., Huneault M. A.: Effect of nucleation and plasticization on the crystallization of poly(lactic acid). Polymer, 48, 6855-6866 (2007).

DOI: $10.1016 /$ j.polymer.2007.09.020

[24] Běhálek L., Maršálková M., Lenfeld P., Habr J., Bobek J., Seidl M.: Study of crystallization of polylactic acid composites and nanocomposites with natural fibres by DSC method. in 'Proceedings of NANOCON 2014, Brno, Czech Republic’ p.6 (2013).

[25] Miyata T., Masuko T.: Crystallization behaviour of poly(L-lactide). Polymer, 39, 5515-5521 (1998). DOI: $10.1016 / \mathrm{S} 0032-3861(97) 10203-8$

[26] Ke T., Sun X.: Melting behavior and crystallization kinetics of starch and poly(lactic acid) composites. Journal of Applied Polymer Science, 89, 1203-1210 (2003). DOI: 10.1002/app.12162 